\title{
Special Issue on Artificial Immune Systems
}

\author{
Peter J. Bentley • Doheon Lee
}

Published online: 21 February 2009

(C) Springer Science + Business Media B.V. 2009

There are many desirable features of natural systems: adaptability, robustness, homeostasis, memory, immunity. Biological immune systems seem to exhibit all of these features and more. Thus it is not so surprising that a vigorous research field has emerged, which focuses on understanding biological immune systems and creating new models, algorithms, technologies and theoretical understandings. The field is known collectively as Artificial Immune Systems, and comprises a remarkably diverse range of researchers. Biologists join forces with mathematicians to create new models. Engineers and computer scientists produce new autonomous intelligent software. Roboticists and specialists in unconventional computation create new control systems or new ways to compute.

In 2008 the International Conference on Artificial Immune Systems moved to East Asia for the first time, not only being held in Thailand with Thai local chairs, but also with conference chairs from South Korea. As its organisers, we were honoured to have such a variety of innovative and original scientific papers. This special issue on Artificial Immune Systems represents a selection of extended versions of the best and most innovative papers from ICARIS 2008.

The first paper, An Artificial Immune System for Clustering Amino Acids in the Context of Protein Function Classification by Secker et al., investigates the representation of proteins and prediction of protein function using a new version of the immune-inspired algorithm opt-aiNet.

\footnotetext{
P. J. Bentley $(\varangle)$

Department of Computer Science, University College London, Malet Place, London WC1E 6BT, UK

e-mail: p.bentley@cs.ucl.ac.uk

D. Lee

Bio-Information System Laboratory, Department of Bio and Brain Engineering, Korea Advanced Institute of Science and Technology, 73-1 Guseong-dong, Yuseong-gu, Daejeon 305-701, South Korea e-mail:dhlee@kaist.ac.kr
} 
Representation in the (Artificial) Immune System by McEwan and Hart continues the theme of representation, but examines the subject from a fresh perspective of computational and statistical learning in an attempt to compensate for what they identify as a low-level inferiority of the shape-space used in current AIS.

Multi-objective Bayesian Artificial Immune System: Empirical Evaluation and Comparative Analyses by Castro and Von Zuben examines the use of an immuneinspired algorithm for combinatorial multiobjective optimization, focusing on the use of a Bayesian network to implement a probabilistic model in order to generate new antibodies. They compare their approach with other methods and show that the Pareto front can be generated with minimal computation time.

Multi-Objective Biclustering: When Non-dominated Solutions are not Enough by Coelho et al., continues the theme of multiobjective optimization, this time focusing on the generation of multiple non-dominated fronts of solutions in parallel, aiming to represent different regions of the original data in a more effective manner than existing methods.

Metabolic Systemic Computing: Exploiting Innate Immunity within an Artificial Organism for On-line Self-Organisation and Anomaly Detection by Le Martelot and Bentley focuses on understanding computation in natural and artificial systems, showing that the tissue and innate immune system of an organism may behave like an anomaly detection system as the organism eats data, builds its internal cells and expels waste.

Finally, AIS based Distributed Wireless Sensor Network For Mobile Search and Rescue Robot Tracking by Ko et al., demonstrates one of the many highly practical uses for immune-inspired algorithms. Here they describe the development of an AIS-based control framework on a distributed wireless sensor network for tracking search and rescue robots.

As exemplified by the work in this special issue, the field of Artificial Immune Systems continues to grow with exciting new theoretical work and practical applications. There is also a constant stream of new discoveries by immunobiologists, so it seems likely that AIS researchers will be active for many years to come.

Peter J. Bentley

Department of Computer Science, UCL

Doheon Lee

Department of Bio and Brain Engineering, KAIST

(Special Issue Editors) 